\title{
Caracterización molecular de carbapenemasas en bacilos gramnegativos circulantes en hospitales de Paraguay. Primer cuatrimestre 2021.
}

\author{
Nancy Melgarejo Touchet ${ }^{(D)}{ }^{1}$, Cristina Mariel Brítez ${ }^{(D)}$, Sofía Busignani ${ }^{(D)}$, Miryan \\ Falcón (iD) 1, Evelyn López ${ }^{(D)}$, Marcela Laconich ${ }^{3}$, Raquel Blasco ${ }^{4}$, Rocío \\ Arguello (iD) 5 , Anibal Kawabata (D)6, Martin Olmedo (iD) 7, Carolina Rojas (D) 8, Marta \\ González ${ }^{1 D}$, Juana Salinas ${ }^{10}$, Karina Abreu (iD) 11, Jazmín Pereira ${ }^{12}$, Eva \\ Mereles ${ }^{13}$, Mario Martínez ${ }^{(D)}$
}

\begin{abstract}
${ }^{1}$ Ministerio de Salud Pública y Bienestar Social, Laboratorio Central de Salud Pública, Dpto.
Bacteriología y Micología. Av. Venezuela y Teniente Escurra, Asunción, Paraguay.

${ }^{2}$ Ministerio de Salud Pública y Bienestar Social, Hospital Nacional de Itaugua. Av. Itaugua Guazu, Itaugua, Paraguay.

${ }^{3}$ Instituto de Previsión Social "Hospital Central", Av. Sacramento y Capitán Lombardo, Asunción, Paraguay.

${ }^{4}$ Ministerio de Salud Pública y Bienestar Social. Hospital Regional de Ciudad del Este. Ciudad del Este, Paraguay.

${ }^{5}$ Universidad Nacional de Asunción, Facultad de Ciencias Médicas, Hospital de Clínicas. Avenida Mariscal López y Coronel Cazal, San Lorenzo, Paraguay.

${ }^{6}$ Hospital del Trauma "Dr. Manuel Giani". Av. General Santos y Teodoro Mongelós, Asunción, Paraguay.

${ }^{7}$ Ministerio de Salud Pública y Bienestar Social. Hospital General Pediátrico "Niños de Acosta $\tilde{N} u^{\prime \prime}$. Av. Arnaldo Bacigalupo, San Lorenzo, Paraguay.

${ }^{8}$ Ministerio de Salud Pública y Bienestar Social. Instituto de Medicina Tropical. Av. Venezuela y Florida, Asunción, Paraguay.

${ }^{9}$ Centro Médico Bautista. Av. República Argentina y Campos Cervera, Asunción, Paraguay.

${ }^{10}$ Sanatorio AMSA. Teniente Fariña y Capitán Figari, Asunción, Paraguay.

${ }^{11}$ Meyer Lab. Coronel Irazábal y Mariscal Estigarribia, Asunción, Paraguay.

${ }^{12}$ Sanatorio Migone. Eligio Ayala 1293, Asunción, Paraguay.

${ }^{13}$ Universidad Nacional del Este, Facultad de Ciencias de la Salud, Laboratorio Epidemiológico Regional de Alto Paraná. Calle Andre Moleón, Minga Guazu, Paraguay.
\end{abstract}

Cómo referenciar este artículo/

How to reference this article:
Melgarejo Touchet N, Brítez CM, Busignani S, Falcón $M$, López $E$, Laconich $M$, et al. Caracterización molecular de carbapenemasas en bacilos gramnegativos circulantes en hospitales de Paraguay. Primer cuatrimestre 2021. Mem. Inst. Investig. Cienc. Salud. 2021; 19(2): 49-58

\section{RE S U M E N}

La resistencia a los antimicrobianos (RAM), representa un grave problema por el uso indiscriminado de antimicrobianos de amplio espectro. En nuestro país, durante el primer cuatrimestre del año, se observó un aumento inusual en el número de aislamiento de gérmenes multirresistentes, sobre todo de bacilos gramnegativos, los cuales fueron remitidos al laboratorio de referencia con el objetivo de caracterizar los genes de resistencia a los carbapenemes. Estudio observacional y prospectivo de corte transversal en 456 aislamientos de bacilos gramnegativos provenientes de 11 centros colaboradores de la Red Nacional de Vigilancia de la RAM, remitidos al Laboratorio Central de Salud Pública entre enero y abril de 2021, para la detección molecular (reacción en cadena de la polimerasa múltiple) de los genes de resistencia enzimática

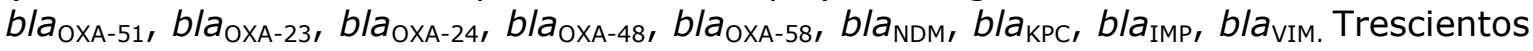
sesenta correspondieron a bacilos gramnegativos no fermentadores: 346 
Acinetobacter baumannii y 14 Pseudomonas aeruginosa; 96 fueron miembros de Enterobacterales, siendo prevalente Klebsiella pneumoniae (81). Todos los aislamientos de Acinetobacter baumannii resultaron ser productores de carbapenemasas: OXA-23 (94\%), NDM (4\%), NMD+OXA-58 (2\%); en Pseudomonas aeruginosa, 7 de los 14 aislamientos (50\%) fueron portadores de metalobetalactamasa del genotipo NDM (100\%). Los genotipos NDM (92\%) y KPC (8\%) fueron confirmados en Enterobacterales. La resistencia plasmídica a carbapenemes es endémica en nuestro país, siendo prevalentes los genotipos OXA-23 en Acinetobacter baumannii y NDM en Pseudomonas aeruginosa y Enterobacterales.

Palabras clave: Resistencia a fármacos, bacteriana, Carbapenemasa, Reacción en Cadena de la Polimerasa, genotipo, Acinetobacter baumannii, Pseudomonas aeruginosa, Enterobacterales, Paraguay.

\title{
Molecular characterization of carbapenemases in Gram-negative bacilli circulating in hospitals of Paraguay. First quarter of 2021.
}

\begin{abstract}
A B S T R A C T
Antimicrobial resistance (AMR) represents a serious problem due to the indiscriminate use of broad-spectrum antimicrobials. During the first quarter of the year, an unusual increase in the number of isolation multi-resistant germs, especially gram-negative bacilli was observed, specially of Gram-negative bacilli which were referred to the reference laboratory in order to characterize the carbapenems resistance genes. Observational and prospective cross-sectional study in 456 isolates of Gram-negative bacilli from 11 collaborating centers of the National AMR Surveillance Network, referred to the Central Public Health Laboratory (LCSP) between January and April 2021, for molecular detection (multiple polymerase chain reaction) targeting the

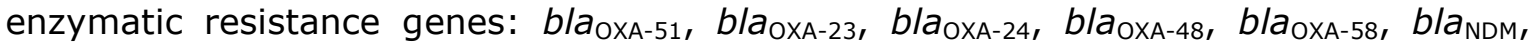
$b / a_{\mathrm{KPC}}, b / a_{\mathrm{IMP}}, b / a_{\mathrm{VIM}}$. Of the 456 isolates studied, 360 corresponded to non-fermenting Gram-negative bacilli, of which 346 were confirmed as Acinetobacter baumannii and 14 Pseudomonas aeruginosa; 96 were Enterobacterales, being Klebsiella pneumoniae (81) the most prevalent. All isolates of Acinetobacter baumannii carried genes encoding carbapenemases, being the OXA-23 (94\%) followed by NDM (4\%) and NDM +OXA-58 (2\%). In Pseudomonas aeruginosa strains, 7 of the 14 isolates (50\%) were carriers of NDM metallobetalactamase $(100 \%)$. No carbapenemase gene was detected in the remaining 7. In all Enterobacterales strains, the presence of carbapenemases of the NDM (92\%) and KPC (8\%) genotypes were confirmed. Plasmid resistance to carbapenems is endemic in our country, being the OXA-23 genotypes prevalent in Acinetobacter baumannii and NDM in Pseudomonas aeruginosa and Enterobacterales.
\end{abstract}

Keywords: antibacterial drug resistance, carbapenemase, Polymerase Chain Reaction, Genotype, Acinetobacter baumannii, Pseudomonas aeruginosa, Enterobacterales, Paraguay.

\section{INTRODUCCIÓN}

Las infecciones asociadas a la atención de la salud (IAAS) generan grandes preocupaciones en todo el mundo, debido a la amplia diseminación de la resistencia a diferentes antimicrobianos de elección, agotando las opciones terapéuticas para el tratamiento de las enfermedades infecciosas, con la consecuente elevada mortalidad e incremento significativo de los costos de atención ${ }^{(1,2)}$.

La resistencia a los antimicrobianos (RAM) es una pandemia de lenta evolución y estrechamente relacionada a las IAAS, que precede a la pandemia ocasionada por el SARS-CoV-2 y continuará aun después de ésta; representando para el futuro un problema de gran índole por el uso inapropiado e indiscriminado de los antimicrobianos de amplio espectro en la profilaxis de las co-infecciones en pacientes internados por COVID-19 $9^{(3,4)}$. 
En bacilos gramnegativos, la resistencia a los carbapenémicos está dada principalmente por la producción de carbapenemasas, ampliamente diseminadas en bacterias de importancia clínica, de diferentes géneros y especies, tales como Acinetobacter spp., Pseudomonas spp. y Enterobacterales. Estas enzimas son variadas y se clasifican según el sustrato utilizado para su detección en los laboratorios de microbiología ${ }^{(5-7)}$.

La primera carbapenemasa detectada fue Klebsiella pneumoniae carbapenemasa (KPC) en el año 1996 en Carolina del Norte, Estados Unidos ${ }^{(8)}$. Posterior a este hallazgo, varios países en diferentes partes del mundo, y en la región, han comunicado la detección de esta enzima ${ }^{(9-13)}$. Paraguay, en el año 2009 reportó su primer hallazgo en aislamientos de Enterobacter sp. ${ }^{(14)}$. Hasta la fecha, en el país sólo se ha confirmado la presencia de KPC en miembros de Enterobacterales ${ }^{(15-17)}$, a diferencia de países vecinos como Argentina y Brasil que la han descrito en Pseudomonas aeruginosa ${ }^{(18,19)}$.

La carbapenemasa del tipo metalobetalactamasa (Clase B), genotipo NDM, fue identificada por primera vez en el año 2008 en un aislado de Klebsiella pneumoniae recuperado de un paciente sueco con antecedente de internación en la India ${ }^{(20)}$. A partir de dicha publicación, la misma fue confirmada en varios países del mundo, principalmente en Enterobacterales ${ }^{(21)}$. En América Latina, Guatemala fue el primer país en notificar esta carbapenemasa en aislamientos de Klebsiella pneumoniae el año 2011, por lo que la Organización Panamericana de la Salud emitió una alerta regional el 22 de noviembre de $2011^{(22)}$. En el año 2014, dicha alerta fue actualizada, ya que varios países de la región (Colombia, Paraguay, Uruguay, Argentina, Brasil, Honduras, México, Nicaragua, Costa Rica) notificaron aislamientos, e incluso brotes ocasionadas por bacterias productoras de dicha carbapenemasa ${ }^{(23)}$.

En Paraguay fue detectada en el año 2012 en aislamientos de Acinetobacter pittii $^{(24,25)}$ y en Enterobacterales, específicamente en Escherichia coli, en el 2015. En la actualidad, este genotipo es de gran diseminación a nivel nacional, en patógenos de importancia clínica, como Pseudomonas aeruginosa, Acinetobacter baumannii y Enterobacterales ${ }^{(26)}$.

A nivel regional, los países han confirmado la presencia de varias familias de estas enzimas (Clase A, B y D) en proporciones diversas ${ }^{(27-30)}$.

La vigilancia laboratorial es fundamental para la detección de estos mecanismos inusuales y emergentes de resistencia. En Paraguay, esta vigilancia tuvo sus inicios en el año 1996 con la conformación de la Red Nacional de Vigilancia de la RAM, coordinada por el Laboratorio Central de Salud Pública (LCSP); desde entonces, todos los hallazgos de importancia fueron detectados por los centros colaboradores, y remitidos al LCSP para la confirmación fenotípica y molecular.

Teniendo en cuenta la situación referida anteriormente y con el objetivo de caracterizar molecularmente las carbapenemasas en los bacilos gramnegativos circulantes en hospitales de Paraguay en el primer cuatrimestre del año 2021, se realizó el presente trabajo.

\section{MATERIALES Y MÉTODOS}

Estudio prospectivo, observacional, descriptivo de corte transversal realizado en aislamientos de bacilos gramnegativos provenientes de 11 centros colaboradores de la Red Nacional de Vigilancia de la RAM, remitidos al LCSP entre enero y abril de 2021, para la confirmación de portación de carbapenemasas, y su genotipificación.

Los criterios de derivación de las cepas fueron:

En Enterobacterales:

- Halo de inhibición de imipenem $\leq 22 \mathrm{~mm}$, o

- Concentración inhibitoria mínima (CIM) en equipo automatizado (Vitek®2 C) de imipenem $\geq 2 \mathrm{ug} / \mathrm{mL}^{(31)}$.

En Pseudomonas aeruginosa:

- Halos de inhibición de ceftazidima $\leq 22 \mathrm{~mm}$ y meropenem $\leq 23 \mathrm{~mm}$, o

- $\mathrm{CIM}$ en equipo automatizado (Vitek $(2 \mathrm{C}$ ) de imipenem $\geq 2 \mathrm{ug} / \mathrm{mL}$, meropenem $\geq 1 \mathrm{ug} / \mathrm{mL}$ y ceftazidima $\geq 16 \mathrm{ug} / \mathrm{mL}^{(32)}$. 
En Acinetobacter spp:

- Halo de inhibición de imipenem $\leq 21 \mathrm{~mm}$ o meropenem $\leq 18 \mathrm{~mm}$, o

- CIM en equipo automatizado (Vitek $\circledast 2$ C) de imipenem $\geq 4 \mathrm{ug} / \mathrm{mL}^{(33)}$.

Fueron estudiados un total de 456 aislamientos remitidos, 360 correspondieron a bacilos gramnegativos no fermentadores y 96 a fermentadores. Las identificaciones de las mismas fueron realizadas por pruebas bioquímicas manuales, automatizadas Vitek ${ }^{\circledR} 2$ Compact (BioMérieux, Francia) y genotípicas (detección de bla OXA-51-like en Acinetobacter spp.).

Tamizaje fenotípico de la producción de carbapenemasas: Fue realizado por el test colorimétrico rápido blue carba ${ }^{(34)}$, y las sinergias entre los discos de carbapenemes con los de ácido fenilborónico (APB) y ácido etilendiaminotetraacético/ácido mercaptoacético de sodio (EDTA/SMA).

Estudio genotípico para la detección de genes de resistencia: Realizado por reacción en cadena de la polimerasa (PCR) múltiple de punto final. Para la detección

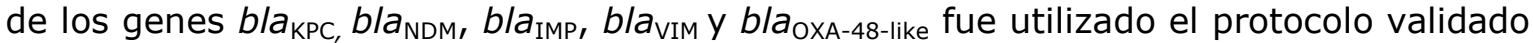
por el Laboratorio Regional de Referencia INEI-ANLIS Dr. Carlos G. Malbrán de Argentina ${ }^{(35)}$; y para la detección de los genes bla ${ }_{\text {OXA-like }}$ (b/aXA-51, bla $a_{\text {OXA-23, }}$ b/a $a_{\text {OXA-24, }}$ bla $\left.a_{\text {OXA-58 }}\right)$ en Acinetobacter spp. fue utilizado el protocolo validado por Woodfor Neil y colaboradores ${ }^{(36)}$. Para la obtención del ADN se utilizó el método de lisis bacteriana por ebullición durante 10 minutos de una suspensión bacteriana de aproximadamente 0,5 Mac Farland en 300 uL de agua libre de RNAsa y centrifugada posteriormente a $10.000 \mathrm{rpm}$ por 10 minutos Las reacciones de amplificación de los genes se realizaron en un termociclador TC-PRO (BOECO Germany) y los productos de amplificación se analizaron mediante electroforesis en gel de agarosa al $2 \%$ en tampón TAE buffer (PanReac AppliChem - ITW Reagents). Las imágenes de los patrones electroforéticos fueron obtenidas con el equipo fotodocumentador Gel Doc ${ }^{\mathrm{TM}}$ EZ Imager (BIO-RAD) y analizadas con el programa Image Lab 6.0 (BIO-RAD).

\section{RESULTADOS}

De los 456 bacilos gramnegativos, 346 fueron confirmados como Acinetobacter baumannii a través de la detección del gen blaXX-51-like, 14 como Pseudomonas aeruginosa y 96 miembros de Enterobacterales.

Los resultados moleculares de los distintos grupos bacterianos fueron:

- En el $100 \%$ de las cepas de Acinetobacter baumannii confirmadas ( $n: 346)$ fueron detectados genes de resistencia enzimática a los carbapenemes: bla OxA$23(94 \%)$, bla $a_{\mathrm{NDM}}(4 \%)$ y la combinación de bla $a_{\mathrm{OXA}-58}$ y bla $\mathrm{NDM}_{\mathrm{NDM}}(2 \%)$.

- En el $50 \%$ de las cepas de Pseudomonas aeruginosa (n:7) fueron detectados los genes de resistencia a los carbapenemes bla $a_{\mathrm{NDM}}$. Las 7 cepas restantes fueron negativas para la portación de carbapenemasas.

- En el $100 \%$ de las cepas de Enterobacterales ( $n$ : 96) fueron detectados los genes de resistencia a carbapenemes: bla $a_{\mathrm{NDM}}(92 \%)$ y $b / a_{\mathrm{KPC}}(8 \%)$.

Los resultados de los estudios fenotípicos y genotípicos se detallan en la Tabla 1.

Tabla 1. Aislamientos de bacilos gramnegativos remitidos al LCSP para estudios de caracterización molecular de carbapenemasas. Enero a abril de 2021. Paraguay. N: 456.

\begin{tabular}{lll}
\hline Grupo bacteriano & $\begin{array}{l}\text { Identificación del } \\
\text { microorganismo }\end{array}$ & $\begin{array}{l}\text { Genotipo de } \\
\text { carbapenemasa detectado }\end{array}$ \\
\hline & $\begin{array}{l}\text { Acinetobacter baumannii (aba) } \\
\text { (n: 346) }\end{array}$ & $\begin{array}{l}\text { OXA-23 }(94 \%) \\
\text { NDM }(4 \%)\end{array}$ \\
NDM + OXA-58 (2\%) \\
$\begin{array}{l}\text { Bacilos gramnegativos no } \\
\text { fermentadores (BGN-NF) } \\
\text { (n: } 360)\end{array}$ & $\begin{array}{l}\text { OXA-51 (100\%) } \\
\text { Pseudomonas aeruginosa (pae) } \\
\text { (n: 14) }\end{array}$ & $\begin{array}{l}\text { NDM }(50 \%) \\
\text { *Negativo (50\%) }\end{array}$ \\
\hline
\end{tabular}




\begin{tabular}{|c|c|c|}
\hline $\begin{array}{l}\text { Enterobacterales (ETB) } \\
(\mathrm{n}: 96)\end{array}$ & $\begin{array}{l}\text { Klebsiella pneumoniae (kpn) } \\
\text { (n: } 81) \\
\text { Enterobacter cloacae (ecl) } \\
\text { (n: 4) } \\
\text { Escherichia coli (eco) } \\
(\mathrm{n}: 3) \\
\text { Klebsiella oxytoca (kox) } \\
(\mathrm{n}: 2) \\
\text { Serratia marcescens (sma) } \\
(\mathrm{n}: 2) \\
\text { Klebsiella aerogenes(eae) } \\
(\mathrm{n}: 1) \\
\text { Citrobacter freundii (cfr) } \\
(\mathrm{n}: 1) \\
\text { Citrobacter diversus(cdi) } \\
(\mathrm{n}: 1) \\
\text { Providencia stuartii (pst) } \\
(\mathrm{n}: 1)\end{array}$ & $\begin{array}{l}\text { NDM }(92 \%) \\
\text { KPC }(8 \%)\end{array}$ \\
\hline
\end{tabular}

En cuanto al origen de los aislamientos:

- Los bacilos gramnegativos no fermentadores ( $A$. baumannii y $P$. aeruginosa) fueron recuperados mayoritariamente de muestras respiratorias (64\%); además de sangre $(13 \%)$, catéter $(9 \%)$, secreciones $(8 \%)$, entre otras.

- Los miembros de Enterobacterales fueron recuperados de muestras de orina $(32 \%)$, muestras respiratorias $(27 \%)$, sangre $(20 \%)$, secreciones $(9 \%)$, catéter $(6 \%)$, entre otras.

\section{DISCUSIÓN}

Las enzimas con acción hidrolítica sobre los carbapenémicos; antimicrobianos de amplio espectro para el tratamiento de las enfermedades infecciosas ocasionadas por gérmenes resistentes a múltiples drogas; se encuentran ampliamente distribuidas en nuestro país, tanto en bacilos gramnegativos fermentadores como no fermentadores. Este año, los centros colabores de la Red Nacional de Vigilancia de la RAM han reportado incremento en el número de aislamiento de cepas con esas características, remitiendo un gran número de las mismas al LCSP para la confirmación de estos mecanismos de resistencias inusuales.

Las cepas de $A$. baumannii remitidas bajo los criterios de sospecha de portación de carbapenemasas, resultaron positivas para la misma en su totalidad (100\%), siendo mayoritariamente oxacilinasas del genotipo OXA-23 (94\%), encontrándose en menor cantidad la metalobetalactamasa genotipo NDM (4\%) y la asociación de la metalobetalactamasa NDM con la oxacilinasa OXA-58 (2\%). Además, fue detectada en

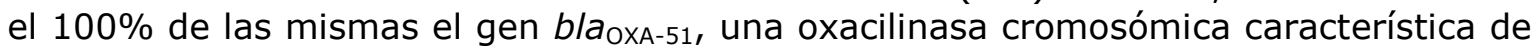
A. baumannii. Varios investigadores han reportado de manera coincidente que OXA-23 es la carbapenemasa prevalente en esta especie bacteriana en varios países de la región. En el año 2016, Rodríguez $\mathrm{CH}$ y colaboradores publicaron los resultados de un trabajo llevado a cabo en cepas aisladas en varios países de Latinoamérica; siendo las correspondientes a Paraguay, recuperadas en un centro en el periodo 2013-2014, productoras de carbapemasa OXA-23 en el $100 \%$. Sin embargo, en el mismo reporte fueron publicados hallazgos de otros genotipos de oxacilinasas y metalobetalactamasa NDM en otros países que participaron del estudio ${ }^{(37,38)}$.

En nuestro país, la carbapenemasa NDM fue reportada por primera vez en Acinetobacter pittii en el año $2012^{(24,25)}$; sin embargo, en $A$. baumannii recién fue confirmada en el año $2015^{(39)}$.

En $P$. aeruginosa, el número de aislamientos sometidos al estudio fue bajo, pudiendo ello generar sesgos en los resultados obtenidos. $50 \%$ de los aislamientos resistentes a carbapenemes resultaron ser portadores de carbapenemasa, de los 
cuales en su totalidad (100\%) del tipo metalobetalactamasa genotipo NDM y $50 \%$ de las cepas restantes, fueron negativas para la portación de carbapenemasas (todas ellas con resultados negativos para las pruebas colorimétricas de blue carba test y sinergias con inhibidores) con características fenotípicas de resistencia debida a la interacción de mecanismos como impermeabilidad y eflujo, ampliamente descritas a nivel regional y mundial ${ }^{(40,41)}$. Hasta la fecha no hemos encontrado los genotipos SPM y KPC reportados por los países vecinos como Brasil y Argentina ${ }^{(19,42,43)}$.

Publicaciones realizadas por varios investigadores de la región revelan las diferencias epidemiológicas entre los países en cuanto a los genes de resistencia diseminados en elementos móviles en P. aeruginosa: en el año 2014, Rizek C. y colaboradores han publicado resultados de un trabajo llevado a cabo en Brasil (bla (32\%) sobre bla $a_{\text {KPC }}$ y bla $a_{\text {VIM }}(3,9 \%)$ ), en el que reportaron incluso coproducción de carbapenemasas (SPM-1 y KPC) ${ }^{(19)}$; sin embargo, resultados muy diferentes han obtenido Mayta-Barrios y colaboradores en aislamientos del año 2019 en Perú, en los que la carbapenemasa mayoritaria en $P$. aeruginosa fue la metalobetalactamasa del genotipo $\operatorname{IMP}(17,3 \%)$ y la minoritaria la $\operatorname{NDM}(1,1 \%)^{(44)}$.

En Enterobacterales, la carbapenemasa del tipo metalobetalactamasa genotipo NDM (96\%) ha desplazado a la primera carbapenemasa detectada en el país (KPC) en el año 2009(14,15); hallazgo coincidente con lo publicado en el 2019 por Martínez M. y colaboradores de un estudio llevado a cabo en aislamientos de 10 años (2009-2019) en un centro hospitalario de la capital del país ${ }^{(45)}$.

Desde la confirmación del genotipo NDM en E. coli por el laboratorio de referencia en el año 2015, hemos detectado este genotipo en varios miembros de Enterobacterales en los diferentes nosocomios del país, siendo $K$. pneumoniae el agente prevalente.

Recientemente, en abril de este año, el Laboratorio de Referencia Regional "Dr. Carlos Malbrán". INEI. ANLIS, emitió una Alerta Epidemiológica: Emergencia de dobles productores de carbapenemasa $\mathrm{KPC}+\mathrm{MBL}^{\prime}$, en la que se describe la confirmación en la primera ola de la pandemia por COVID-19 la emergencia y diseminación de colonización/infección de Enterobacterales productores de combinaciones de carbapenemasas en la Argentina ${ }^{(46)}$. Publicaciones de hallazgos de coproducción de carbapenemasas en $K$. pneumoniae fueron realizadas por investigadores de Brasil, como Nava RG y colaboradores en el 2019(47); y, Oliveira EM y colaboradores en el $2020^{(48)}$. Estos hallazgos dificultan aún más el tratamiento de las enfermedades infecciosas, teniendo en cuenta los diferentes fármacos de elección para los distintos genotipos. En Paraguay, Rivas M y colaboradores en un estudio llevado a cabo en el 2017 en un centro hospitalario, con bacilos gramnegativos resistentes a los carbapenemes, reportaron la coproducción en 1 de 101 cepas estudiadas, sin referir a que grupo ni especie bacteriana correspondía ${ }^{(49)}$. En este estudio, no hemos encontrado la doble producción de carbapenemasas en Enterobacterales.

La epidemiología de las carbapenemasas es muy variable, observándose grandes diversidades según regiones, países, nosocomios y en constante cambio a través del tiempo, por la gran capacidad de diseminación en elementos genéticos móviles. Ello constituye un permanente desafío para el control de las enfermedades infecciosas, ya que los microorganismos son capaces de albergar y combinar genes de resistencia a carbapenémicos, incluso asociados a otros genes de resistencia a diferentes familias de antibióticos.

La detección oportuna de estos mecanismos de resistencia de rápida diseminación constituye uno de los pilares fundamentales para la contención de la propagación de los mismos; por lo que es fundamental proveer de capacidades técnicas y logísticas a los laboratorios de Microbiología.

\section{CONTRIBUCIÓN DE AUTORES}

Melgarejo Touchet Nancy: Autor principal. Idea, Elaboración del Protocolo, recolección y análisis de los datos, presentación de resultados, redacción del manuscrito.

- Martínez Mario: Asesoría, revisión crítica del manuscrito. 
- Brítez Mariel: Estudios fenotípicos y genotípicos completos de los aislamientos bajo investigación.

- Busignani Sofía: Estudios fenotípicos y genotípicos completos de los aislamientos bajo investigación.

- Falcón Miryan, López Evelyn, Laconich Marcela, Blasco Raquel, Arguello Rocío, Kawabata Anibal, Olmedo Martin, Rojas Carolina, González Marta, Salinas Juana, Abreu Karina, Pereira Jazmín, Mereles Eva: Detección fenotípica de resistencias inusuales en las cepas de interés; y remisión de las mismas al Laboratorio de Referencia.

\section{REFERENCIAS BIBLIOGRÁFICAS}

1. Cardo D, Dennehy $\mathrm{PH}$, Halverson $\mathrm{P}$, Fishman N, Kohn M, Murphy $\mathrm{CL}$, et al. Moving toward elimination of healthcare associated infections: a call to action. Infect Control Hosp Epidemiol. 2010 Nov; 31(11): 1101-5. 10.1086/656912. PMID: 20929300.

2. Pujol M, Limón E. Epidemiología general de las infecciones nosocomiales. Sistemas y programas de vigilancia [General epidemiology of nosocomial infections. Surveillance systems and programs]. Enferm Infecc Microbiol Clin. 2013 Feb; 31(2): 108-13.

Doi: 10.1016/j.eimc.2013.01.001. PMID: 23357654.

3. Pérez-Lazo G, Soto-Febres F, MoralesMoreno A, Cabrera-Enríquez JA, DíazAgudo J, Rojas R, et al. Uso racional de antimicrobianos en tiempos de COVID-19 en Perú: rol de los programas de optimización del uso de antimicrobianos e intervenciones desde el punto de vista de control de infecciones. Horizonte Médico (Lima). 2021; 21(2): 1254.

Doi: 10.24265/horizmed.2021.v21n2.12.

4. Bengoechea JA, Bamford CG. SARS-CoV2, bacterial co-infections, and AMR: the deadly trio in COVID-19? EMBO Mol Med. 2020 Jul 7; 12(7): e12560. doi: 10.15252/emmm.202012560. Epub 2020 Jun 15. PMID: 32453917; PMCID: PMC7283846.

5. Livermore DM. The impact of carbapenemases on antimicrobial development and therapy. Curr Opin Investig Drugs. 2002 Feb; 3(2): 218-24. PMID: 12020049.

6. Bush K, Jacoby GA. Updated functional classification of beta-lactamases. Antimicrob Agents Chemother. 2010 Mar; 54(3): 969-76. doi: 10.1128/AAC.0100909. Epub 2009 Dec 7. PMID: 19995920; PMCID: PMC2825993.

7. Ambler RP. The structure of betalactamases. Philos Trans R Soc Lond B Biol Sci. 1980 May 16; 289 (1036): 32131. doi: 10.1098/rstb.1980.0049. PMID: 6109327.

8. Yigit $H$, Queenan AM, Anderson GJ, Domenech-Sanchez A, Biddle JW,
Steward CD, et al. Novel carbapenemhydrolyzing beta-lactamase, KPC-1, from a carbapenem-resistant strain of Klebsiella pneumoniae. Antimicrob Agents Chemother. 2001 Apr; 45(4): 1151-61. doi: 10.1128/AAC.45.4.1151-1161.2001. Erratum in: Antimicrob Agents Chemother. 2008 Feb; 52(2):809. PMID: 11257029; PMCID: PMC90438.

9. Hossain A, Ferraro MJ, Pino RM, Dew III RB, Moland ES, Lockhart TJ, et al. Plasmid-mediated carbapenemhydrolyzing enzyme KPC-2 in an Enterobacter sp. Antimicrob. Agents Chemother.2004; 48: 4438-40.

10. Villegas MV, Lolans K, Correa A, Suarez CJ, Lopez JA, Vallejo M, Quinn JP. Colombian Nosocomial Resistance Study Group. First detection of the plasmidmediated class A carbapenemase KPC-2 in clinical isolates of Klebsiella pneumoniae from South America. Antimicrob Agents Chemother. 2006 Aug; 50 (8): 2880-2. 10.1128/AAC.00186-06. PMID: 16870793; PMCID: PMC1538657

11. Bradford PA, Bratu S, Urban C, Visalli M, Mariano N, Landman D, et al. Emergence of carbapenem-resistant Klebsiella species possessing the class $A$ carbapenem-hydrolyzing KPC-2 and inhibitor-resistant TEM-30 betalactamases in New York City. Clin Infect Dis. 2004 Jul 1; 39(1): 55-60. doi: 10.1086/421495. Epub 2004 Jun 14. PMID: 15206053.

12. Pasteran FG, Otaegui L, Guerriero L, Radice G, Maggiora R, Rapoport M, et al. Klebsiella pneumoniae Carbapenemase-2, Buenos Aires, Argentina. Emerg Infect Dis. $2008 \mathrm{Jul} ;$ 14(7):1178-80.

doi: 10.3201/eid1407.070826. PMID: 18598660; PMCID: PMC2600346.

13. Monteiro J, Santos AF, Asensi MD, Peirano G, Gales AC. First report of KPC2-producing Klebsiella pneumoniae strains in Brazil. Antimicrob Agents Chemother. 2009 Jan; 53(1): 333-4. doi: 10.1128/AAC.00736-08. Epub 2008 Nov 17. PMID: 19015350; PMCID: PMC2612176. 
14. Melgarejo N, Martínez M, Franco R, Lird G, Laconich M, Aguilar G. Detección de carbapenemasa (KPC) en Enterobacter en un hospital de Asunción, Paraguay. VII Congreso paraguayo de Infectología. I Jornada de Microbiología Clínica. V Jornada de Enfermería. 6, 7, 8 de noviembre de 2009.

15. Melgarejo N, Martínez M, Franco $R$, Falcón M. Enterobacterias resistentes a Carbapenemes por producción de KPC, aisladas en hospitales de Asunción y Departamento Central. Revista de Salud Pública del Paraguay. 2013; 3(1): 30-5.

16. Dirección General de Vigilancia de la Salud. Ministerio de Salud Pública y Bienestar Social. Alerta Epidemiológica. Primer hallazgo de Metalobetalactamasa. New Delhi (NDM) en Paraguay. 26 de noviembre de 2012. Disponible en:

https://www.mspbs.gov.py/dependencias /imt/uploads/Documento/alerta6.pdf.

17. Melgarejo TN, Martínez $M$, Franco $R$, Falcón M, Álvarez M, Ortiz $H$, Irala J. Primer aislamiento de Salmonella Javiana con portación de KPC-2 en Paraguay. Rev. salud publica Paraguay. 2017 Dic; 7(2): 51-6. Disponible en: http://scielo.iics.una.py/scielo.php?script =sci abstract\&pid=S2307-

33492017000200051\&lng=es\&nrm=iso\&t Ing=es.

18. Scavuzzi AML, Beltrão EMB, Firmo EF, de Oliveira ÉM, Beserra FG, Lopes ACS. Emergence of bla ${ }_{\mathrm{VIM}-2}, b / a_{\mathrm{NDM}-1}, b / a_{\mathrm{IMP}-7}$ and bla $a_{\mathrm{GES}-1}$ in bla $\mathrm{KPC}-2$-harbouring Pseudomonas aeruginosa isolates in Brazil. J Glob Antimicrob Resist. 2019 Dec; 19: 181-2. doi: 10.1016/j.jgar.2019.09.009. Epub 2019 Sep 19. PMID: 31542553.

19. Rizek C, Fu L, Dos Santos LC, Leite G, Ramos J, Rossi F, Guimaraes T, Levin AS, Costa SF. Characterization of carbapenem-resistant Pseudomonas aeruginosa clinical isolates, carrying multiple genes coding for this antibiotic resistance. Ann Clin Microbiol Antimicrob. 2014 Sep 2; 13: 43. doi: 10.1186/s12941-014-0043-3. PMID: 25179208 ; PMCID: PMC4282171.

20. Yong D, Toleman MA, Giske CG, Cho HS, Sundman K, Lee K, Walsh TR. Characterization of a new metallo-betalactamase gene, bla(NDM-1), and a novel erythromycin esterase gene carried on a unique genetic structure in Klebsiella pneumoniae sequence type 14 from India. Antimicrob Agents Chemother. 2009 Dec; 53(12): 5046-54. doi: 10.1128/AAC.00774-09. Epub 2009 Sep 21. PMID: 19770275; PMCID: PMC2786356.
21. Dortet $L$, Poirel $L$, Nordmann $P$. Worldwide dissemination of the NDM-type carbapenemases in Gram-negative bacteria. Biomed Res Int. 2014; 2014: 249856. doi: 10.1155/2014/249856. Epub 2014 Mar 26. PMID: 24790993; PMCID: PMC3984790.

22. PAHO. org. Alerta epidemiológica. PAHOWHO Primer hallazgo de carbapenemasa de tipo New Delhi metalobetalactamasa (NDM) en Latinoamérica. 22 de noviembre de 2011. Disponible en: https://www.paho.org/hq/dmdocuments/ 2012/22-noviembre-2011-

carbapenemasas-americas1.pdf.

23. PAHO.org. Actualización Epidemiológica PAHO-WHO. Carbapenemasa de tipo New Delhi metalobetalactamasa (NDM). 7 de marzo de 2014. Disponible en: https://iris.paho.org/bitstream/handle/10 665.2/50690/EpiUpdate7March2014_eng. pdf? sequence $=1$ \&isAllowed $=y$

24. Dirección General de Vigilancia de la Salud. Ministerio de Salud Pública y Bienestar Social. Alerta Epidemiológica. Primer hallazgo de Metalobetalactamasa. New Delhi (NDM) en Paraguay. 26 de noviembre de 2012. Disponible en: https://www.mspbs.gov.py/dependencias Limt/uploads/Documento/alerta6.pdf.

25. Pasteran F, Mora MM, Albornoz E, Faccone D, Franco R, Ortellado J, et al. Emergence of genetically unrelated NDM1-producing Acinetobacter pittii strains in Paraguay. J Antimicrob Chemother. 2014 Sep; 69(9): 2575-8. doi: 10.1093/jac/dku139. Epub 2014 May 3. PMID: 24793901.

26. Rivas $M$, Ortiz H, Almada $P$, Arguello $R$, Melgarejo N, Martínez C. Escherichia coli metalobetalactamasa en un hospital de alta complejidad en Paraguay. Rev. virtual Soc. Parag. Med. Int 2016; 3 (2):120-3.

http://scielo.iics.una.py/scielo.php?script =sci arttext\&pid=S2312-

$\underline{38932016000200011 \& \operatorname{lng}}=$ en\%22lng =en

27. Escandón-Vargas K, Reyes S, Gutiérrez S, Villegas MV. The epidemiology of carbapenemases in Latin America and the Caribbean. Expert Rev Anti Infect Ther. 2017 Mar; 15(3): 277-97. doi: 10.1080/14787210.2017.1268918. Epub 2016 Dec 20. PMID: 27915487.

28. Pasteran F, Corso A, Instituto Nacional de Enfermedades Infecciosas (INEI). ANLIS "Dr. Carlos Malbran". Servicio Antimicrobianos. Programa Nacional de Control de Calidad en Bacteriología. Alerta: diseminación de KPC en Argentina. Buenos Aires: INEI, 2010.

29. Monteiro J, Santos AF, Asensi MD, Peirano G, Gales AC. First report of KPC2-producing Klebsiella pneumoniae 
strains in Brazil. Antimicrob Agents Chemother. 2009 Jan; 53(1): 333-4. doi: 10.1128/AAC.00736-08. Epub 2008 Nov 17. PMID: 19015350; PMCID: PMC2612176.

30. Pavez M, Mamizuka EM, Lincopan N. Early dissemination of KPC-2-producing Klebsiella pneumoniae strains in Brazil. Antimicrob Agents Chemother. 2009 Jun; 53 (6): 2702. doi: 10.1128/AAC.0008909. Epub 2009 Mar 30. Erratum in: Antimicrob Agents Chemother. 2009 Jul; 53(7): 3180. PMID: 19332672; PMCID: PMC2687248.

31. Servicio Antimicrobianos, Instituto Nacional de Enfermedades Infecciosas, Administración Nacional de Laboratorios e Institutos de Salud "Dr. Carlos G. Malbrán". Criterios para sospecha de carbapenemasas en Enterobacterias 2019. Disponible en:

http://antimicrobianos.com.ar/ATB/wpcontent/uploads/2019/10/CRITERIOSPARA-SOSPECHA-DE-CARBAPENEMASASEN-ENTEROBACTERIAS-2019.pdf.

32. Servicio Antimicrobianos, Instituto Nacional de Enfermedades Infecciosas, Administración Nacional de Laboratorios e Institutos de Salud "Dr. Carlos G. Malbrán". Criterios para sospecha de carbapenemasas en $P$. aeruginosa. Disponible en:

http://antimicrobianos.com.ar/ATB/wpcontent/uploads/2020/11/Algoritmo-dedeteccion-de-carbapenemasas-en-P.aeruginosa-2019.pdf.

33. Servicio Antimicrobianos, Instituto Nacional de Enfermedades Infecciosas, Administración Nacional de Laboratorios e Institutos de Salud "Dr. Carlos G. Malbrán". Criterios para sospecha de carbapenemasas en $P$. aeruginosa Disponible en:

http://antimicrobianos.com.ar/ATB/wpcontent/uploads/2018/05/Algoritmoactualizado-para-la-vigilancia-de-MBLKPC-en-Acinetobacter-spp.pdf.

34. Pires J, Novais A, Peixe L. Blue-carba, an easy biochemical test for detection of diverse carbapenemase producers directly from bacterial cultures. J Clin Microbiol. 2013 Dec; 51(12): 4281-3. doi: $10.1128 /$ JCM.01634-13. Epub 2013 Oct 9. PMID: 24108615; PMCID: PMC3838089.

35. Servicio Antimicrobianos, Instituto Nacional de Enfermedades Infecciosas, Administración Nacional de Laboratorios e Institutos de Salud "Dr. Carlos G. Malbrán". Protocolo de PCR multiplex para detección de carbapenemasas. Disponible en:
http://antimicrobianos.com.ar/ATB/wpcontent/uploads/2021/01/Deteccion-CBPMultiplex.pdf.

36. Woodford $\mathrm{N}$, Ellington $\mathrm{MJ}$, Coelho JM, Turton JF, Ward ME, Brown S, Amyes SG, Livermore DM. Multiplex PCR for genes encoding prevalent OXA carbapenemases in Acinetobacter spp. Int J Antimicrob Agents. 2006 Apr; 27(4): 351-3. doi: 10.1016/j.ijantimicag.2006.01.004. Epub 2006 Mar 24. PMID: 16564159.

37. Rodríguez $\mathrm{CH}$, Nastro $\mathrm{M}$, Famiglietti $\mathrm{A}$. Carbapenemases in Acinetobacter baumannii. Review of their dissemination in Latin America. Rev Argent Microbiol. 2018 Jul-Sep; 50(3):327-33. doi: 10.1016/j.ram.2017.10.006. Epub 2018 Mar 13. PMID: 29548732.

38. Rodríguez $\mathrm{CH}$, Balderrama Yarhui $\mathrm{N}$, Nastro M, Nuñez Quezada T, Castro Cañarte G, Magne Ventura $R$, et al. Molecular epidemiology of carbapenemresistant Acinetobacter baumannii in South America. J Med Microbiol. 2016 Oct; 65(10): 1088-91. doi: 10.1099/jmm.0.000328. Epub 2016 Aug 5. PMID: 27498975.

39. Comunicado de Circulación de $P$. aeruginosa y Acinetobacter spp. multirresistentes (incluyendo a colistina) en hospitales de Paraguay. Disponible en: https://www.mspbs.gov.py/dependencias /lcsp/adjunto/2dc110-

ResumenGMRcomunicadoLCSPmayo2018. pdf.

40. Suárez C, Kattán J, Guzmán A, Villegas M. Mecanismos de resistencia a carbapenems en $P$. aeruginosa, Acinetobacter y Enterobacteriaceae y estrategias para su prevención y control. Infect. 2006; 10(2): 85-93. Disponible en:

http://www.scielo.org.co/scielo.php?scrip $\mathrm{t}=\mathrm{sci}$ arttext\&pid=S012393922006000200006\&lng =en\%22Ing=en

41. Orecchini LA, López $T$, Littvik $A$. Resistencia a carbapenemes en Pseudomonas aeruginosa en un periodo de 10 años en el Hospital Rawson [Carbapenems resistance in Pseudomonas aeruginosa during a 10year period at Rawson Hospital]. Rev Fac Cien Med Univ Nac Cordoba. 2010; 67(4): 135-40. Spanish. PMID: 21843437.

42. de Oliveira Santos IC, Albano RM, Asensi MD, D'Alincourt Carvalho-Assef AP. Draft genome sequence of KPC-2-producing Pseudomonas aeruginosa recovered from a bloodstream infection sample in Brazil. J Glob Antimicrob Resist. 2018 Dec; 15: 99-100. doi: 10.1016/j.jgar.2018.08.021. Epub 2018 Aug 30. PMID: 30172833. 
43. Pasteran F, Faccone D, Gomez S, De Bunder S, Spinelli F, Rapoport M, et al. Pseudomonas aeruginosa KPC Group. Detection of an international multiresistant clone belonging to sequence type 654 involved in the dissemination of KPC - producing Pseudomonas aeruginosa in Argentina. J Antimicrob Chemother. 2012 May; 67(5):1291-3. doi: 10.1093/jac/dks032. Epub 2012 Feb 20. PMID: 22354953.

44. Mayta-Barrios MM, Ramirez-Illescas JJ, Pampa-Espinoza L, Yagui-Moscoso MJA. Molecular characterization of carbapenemases in Peru during 2019. Rev. perú. med. exp. salud publica. 2021 Jan-Mar; 38(1): 113-8. Spanish, English. doi: $10.17843 /$ rpmesp.2021.381.5882. Epub 2021 Jun 25. PMID: 34190902.

45. Martínez M, Silvagni M, Vega M, Grau L, Sosa $T$, Espínola $S$, et al. Desplazamiento de la frecuencia del tipo de carbapenemasa en Enterobacterias aisladas en un centro hospitalario de Asunción, 2009 a marzo de 2019, XIX Congreso Panamericano de Infectología. XII Congreso Paraguayo de Infectología, 2019, Asunción-Paraguay. Revista del Instituto de Medicina Tropical. 2019; 14 (suplemento): 52.

46. Alerta Epidemiológica. Emergencia de Enterobacterales dobles productores de carbapenemasa. INEI. ANLIS.
Malbrán.2021. Disponible en:

http://antimicrobianos.com.ar/2021/04/e mergence-of-enterobacterales-with-coexpression-of-two-carbapenemasesduring-covid-19-pandemic-in-argentina/

47. Nava RG, Oliveira-Silva $M$, NakamuraSilva R, Pitondo-Silva A, Vespero EC. New sequence type in multidrug-resistant Klebsiella pneumoniae harboring the blaNDM-1-encoding gene in Brazil. Int J Infect Dis. 2019 Feb; 79: 101-3. doi: 10.1016/j.ijid.2018.11.012. Epub 2018 Nov 26. PMID: 30496852.

48. Oliveira ÉM, Beltrão EMB, Scavuzzi AML, Barros JF, Lopes ACS. High plasmid variability, and the presence of IncFIB, IncQ, IncA/C, IncHI1B, and IncL/M in clinical isolates of Klebsiella pneumoniae with bla KPC and bla NDM from patients at a public hospital in Brazil. Rev Soc Bras Med Trop. 2020 Oct 21; 53: e20200397. doi: 10.1590/0037-86820397-2020.

PMID: 33111914 ; PMCID: PMC7580274.

49. Rivas $M$, Zubeldía A, Cuevas $A$, Ortellado J, Guillén R, Acuña $P$, et al. Detección de genes de resistencia a carbapenemes en bacilos gram negativos aislados de pacientes del hospital de clínicas en el año 2017. XIX Congreso Panamericano de Infectología. XII Congreso Paraguayo de Infectología, 2019, Asunción-Paraguay. Revista del Instituto de Medicina Tropical. 2019; 14 (suplemento): 53. 